\title{
Characterisation of Arabidopsis flotillins in response to stresses
}

\author{
K. KROUMANOVÁ ${ }^{1}$, D. KOCOURKOVÁ ${ }^{1}$, M. DANĚK ${ }^{1,2}$, L. LAMPAROVÁ ${ }^{1,3}$, R. POSPÍCHALOVÁ $^{1}$, \\ K. MALÍNSKÁ ${ }^{1}$, Z. KRČKOVÁ ${ }^{1}$, L. BURKETOVÁ ${ }^{1}$, O. VALENTOVÁ $^{3}$, J. MARTINEC $^{1}$, and M. JANDA ${ }^{1,3 *}$ \\ Institute of Experimental Botany of the Czech Academy of Sciences, CZ-16502, Prague, Czech Republic ${ }^{1}$ \\ Department of Experimental Plant Biology, Faculty of Science, Charles University, \\ CZ-12844 Prague, Czech Republic ${ }^{2}$ \\ Department of Biochemistry and Microbiology, University of Chemistry and Technology, Prague, \\ CZ-16628 Prague, Czech Republic ${ }^{3}$
}

\begin{abstract}
Plant flotillins, a subgroup of the SPFH domain protein superfamily, consist of three proteins, AtFLOT1, AtFLOT2, and AtFLOT3 in Arabidopsis thaliana. The exact functions of flotillins in plant cell has not been established yet. In this study we focused on the role of flotillins in response to both abiotic and biotic stresses and on the response to phytohormones abscisic acid and 1-naphthalene acetic acid (NAA) in A. thaliana. We observed transcriptomic changes of AtFLOT genes in response to high salinity and cold, treatment with 22-amino acid peptide from N-terminal part of flagellin (flg22), and after infection with Botrytis cinerea. Transcription of AtFLOT2 increased up to 60 times after flg22 treatment. Also, treatment with B. cinerea increased transcription of AtFLOT1 10 times and of AtFLOT3 14 times. Furthermore, we used T-DNA knock-out single mutants for all three $A$. thaliana flotillins and we measured root growth in response to high salinity, cold, phosphate starvation, nitrogen starvation, and abscisic acid and NAA treatments. Subsequently, we measured the reactive oxygen species production and callose accumulation after the treatment with flg22. Next, we performed resistance assays to Pseudomonas syringae pv. tomato DC3000 and B. cinerea. In contrast to transcriptomic changes, knocking-out of only single FLOT gene did not lead to significant changes in response to all tested stresses.
\end{abstract}

Additional key words: abscisic acid, auxin, Botrytis cinerea, callose, cold, nutrient starvation, Pseudomonas syringae, ROS, salinity.

\section{Introduction}

Plants evolved sophisticated, efficient, and complex responses to both biotic and abiotic stresses. Plasma membrane (PM) serves as a highly exposed platform for responses to stress factors. Receptors responsible for recognition of threats are often present on PM (Ott 2017). Within PM, the crucial role has its compartmentalization to macro, micro, and nanodomains (Sekeres et al. 2015). It was shown that membrane microdomains are important for membrane trafficking, signal transduction, and response to pathogen attack (Lefebvre et al. 2007, Liu et al. 2009,

Wang et al. 2015, Bucherl et al. 2017).

Plant flotillins along with prohibitins (PHB) belong to the stomatin/prohibitin/flotillin/HflK/C (SPFH) domain (also known as Band 7 domain) protein superfamily. Comparative genome analysis of this superfamily reveals deep evolutionary origin and diverse gene functions (Di et al. 2010). Flotillins are associated with membrane microdomains and are commonly used as markers of membrane microdomains in both mammalian and plant cells. Flotillins occur not only on the PM but also were

Submitted 10 May 2018, last revision 26 June 2018, accepted 30 June 2018.

Abbreviations:ABA - abscisic acid, Col-0 - Columbia-0, dpi - days post inoculation, elf18 - acetylated 18-amino acid fragment from $\mathrm{N}$-terminal of elongation factor Tu (acetyl-MSKEKFERTKPHVNVGT), flg22 - 22-amino acid peptide from N-terminal part of flagellin (QRLSTGSRINSAKDDAAGLQIA), FLS2 - flagellin-sensitive 2, FLOT - flotillin, hpi - hours post inoculation, JA - jasmonic acid, MAMP - microbe-associated molecular pattern, MS - Murashige and Skoog, NAA - 1-naphthalene acetic acid, PM - plasma membrane, Pst - Pseudomonas syringae pv. tomato strain DC3000, ROS - reactive oxygen species, SA - salicylic acid, Ws-4 - Wassilievska-4, WT - wild-type.

Acknowledgments: This research was supported by the Czech Science Foundation (grant No. 14-09685S) and by the Ministry of Education, Youth, and Sport of the Czech Republic (MSMT No 21-SVV/2017).

* Author of correspondence; e-mail: martin.janda@vscht.cz 
detected in endosomes (Glebov et al. 2006, Haney et al. 2010, Li et al. 2012, Jarsch et al. 2014, Yu et al. 2017). Three members of flotillin protein family were identified in A. thaliana, AtFLOT1 (At5g25250), AtFLOT2 (At5g52560), and AtFLOT3 (At5g64870). It should be noted that in some literature the AtFLOT1 and AtFLOT2 are affiliated as $A t$ FLOT1a and $A t$ FLOT1b, respectively (Jarsch et al. 2014). Yu et al. (2017) showed that AtFLOT1 and AtFLOT2 share $94 \%$ similarity of amino acid sequence and AtFLOT1 share $85 \%$ similarity with AtFLOT3.

Flotillin functions were broadly studied and described in yeasts and mammals; while the proper role of flotillins in plants is still very barely understood. In plants flotillins were shown to play important role in plant-microbe interaction. In Medicago truncatula, seven genes encoding flotillin-like proteins were identified (Haney et al. 2010). From those MtFLOT2 and MtFLOT4 were significantly upregulated during early symbiotic events and play crucial role in establishing the relationship between $M$. truncatula and symbiotic nitrogen-fixing rhizobium Sinorhizobium

\section{Materials and methods}

Plants and cultivation: In this study we used Arabidopsis thaliana wild type (WT) genotypes: Columbia-0 (Col-0) and Wassilievska-4 (Ws-4) and mutants Atflot1 (SALK_203966C) and Atflot3 (SALK_143325C) with Col-0 background, and Atflot2 (FLAG_352D08) with Ws4 background.

Surface-sterilized seeds were sown in Jiffy 7 peat pellets and plants were grown for four weeks in soil, under a 10-h photoperiod, an irradiance of $90-120 \mu \mathrm{mol} \mathrm{m}^{-2} \mathrm{~s}^{-1}$, a temperature of $22{ }^{\circ} \mathrm{C}$ and a $70 \%$ relative humidity. They were watered with distilled water as necessary. Plants grown in these conditions were used for reactive oxygen species (ROS) determination and Pst DC3000 and Botrytis cinerea treatments.

Further, A. thaliana seedlings were grown in liquid Murashige and Skoog (MS) medium or on solid $1 / 2 \mathrm{MS}$ medium. The liquid MS medium contained $4.41 \mathrm{~g} \mathrm{dm}^{-3} \mathrm{MS}$ vitamins (Duchefa, Haarlem, The Netherlands), $5 \mathrm{~g} \mathrm{dm}^{-3}$ sucrose, and $5 \mathrm{~g} \mathrm{dm}^{-3}$ (N-morpholino) ethanesulfonic acid (MES) monohydrate (Duchefa). The solid $1 / 2 \mathrm{MS}$ medium contained $2.2 \mathrm{~g} \mathrm{dm}^{-3} \mathrm{MS}$ basal salts (Duchefa) and $10 \mathrm{~g} \mathrm{dm}^{-3}$ agar (Sigma-Aldrich, St. Louis, USA). Both media were adjusted to $\mathrm{pH} 5.8$ using $1 \mathrm{M} \mathrm{KOH}$. For cultivation in the liquid, surface-sterilized seeds were sown in 24-well plates containing $0.4 \mathrm{~cm}^{3}$ of liquid MS medium per well and 3 - 5 seeds. Plants were cultivated for $11 \mathrm{~d}$ under a 10-h photoperiod, an irradiance of $100-130$ $\mu \mathrm{mol} \mathrm{m} \mathrm{m}^{-2} \mathrm{~s}^{-1}$ and a temperature of $22{ }^{\circ} \mathrm{C}$. On the $7^{\text {th }}$ day, the medium in the wells was exchanged for a fresh one. Seedlings from liquid media were used for callose analysis.

For the root length analysis, the seedlings were grown meliloti. Additionally, co-localization of MtFLOT4mCherry with lysin motif receptor-like kinase 3 (LYK3) was observed in inoculated roots (Haney et al. 2010). Yu et al. (2017) showed that treatment of plants with flg22 leads to the increased degradation of AtFLOT1. Moreover, accumulation of callose decreased in Atflot1 amiRNAi plants in response to flg22 (Yu et al. 2017). By contrast, to our best knowledge, no data are available for the role of flotillins in plant responses to abiotic stresses. In sillico transcription analysis performed using Genevestigator ${ }^{\circledR}$. It was shown that gene transcription of AtFLOTs is increased under various abiotic and biotic stresses (Daněk et al. 2016).

The present study was focused on the role of AtFLOTs in response to following treatments: high salinity, cold, nitrogen and phosphate starvation, abscisic acid (ABA), 1-naphthalene acetic acid (NAA), Pseudomonas syringae (Pst) and Botrytis cinerea infection, and elicitors flg22 or elf18. We analysed transcription of AtFLOTs in WT plants together with knock-out T-DNA single mutants of flotillin genes.

on solid medium in square plates $(12 \mathrm{~cm}$ side). The plates with seeds were placed to $4{ }^{\circ} \mathrm{C}$ for $48 \mathrm{~h}$. Then, the seedlings were grown under a 16-h photoperiod, an irradiance of $100 \mu \mathrm{mol} \mathrm{m} \mathrm{m}^{-2} \mathrm{~s}^{-1}$, and a temperature of $22^{\circ} \mathrm{C}$. At $5^{\text {th }}$ day, seedlings of similar size were transferred to new plates and the length of root was marked. Experiments were designed so that WT seedlings and the particular mutant line were on the same plate. In one biological replicate 20 seedlings for WT and 17 - 20 seedlings for particular mutant line were used. At $7^{\text {th }}$ day after transfer, the root length increase was marked. For the 1-naphthalene acetic acid (NAA) treatment, seedlings of similar size were transferred to new plates at $4^{\text {th }}$ day and at $4^{\text {th }}$ day after transfer, the root length increase was marked. The experiments were performed in three biological repeats.

Transcriptomic analysis: For transcriptomic analysis, seedlings were grown on solid $1 / 2$ MS media in round plates (6 $\mathrm{cm}$ in diameter) lying horizontally in a 16-h photoperiod, an irradiance of $100 \mu \mathrm{mol} \mathrm{m} \mathrm{m}^{-2} \mathrm{~s}^{-1}$, and a temperature of $22{ }^{\circ} \mathrm{C}$. The seedlings $(10-12$ from each plate) were harvested at day 11 and the tissue was stored in liquid nitrogen. For one biological replicate 3 - 4 independent samples were used.

Total RNA was isolated from frozen plant tissue using the Spectrum Plant Total RNA kit (Sigma-Aldrich) and treated with a DNA-free kit (Ambion, Austin, TX, USA). Then $1 \mu \mathrm{g}$ of RNA was used for reverse transcription to cDNA with $M-M L V$ RNase $H^{-}$Point Mutant reverse transcriptase (Promega, Fitchburg, WI, USA) and anchored oligo dT21 primer (Metabion, Martinsried, Germany). Gene transcription was quantified by q-PCR 
using LightCycler 480 SYBR Green I Master kit and LightCycler 480 (Roche, Basel, Switzerland). The PCR conditions were: $95{ }^{\circ} \mathrm{C}$ for $10 \mathrm{~min}$, followed by 45 cycles of $95^{\circ} \mathrm{C}$ for $10 \mathrm{~s}, 55^{\circ} \mathrm{C}$ for $20 \mathrm{~s}$, and $72{ }^{\circ} \mathrm{C}$ for $20 \mathrm{~s}$, followed by a melting curve analysis. Relative transcription was calculated with normalization to the housekeeping gene TIP41-like (At4g34270). The list of primers is in Table 1 Suppl.

Abiotic stresses: Under following abiotic stress conditions root length was measured. Seedlings were scanned at day 7 after transfer. Root length was analysed

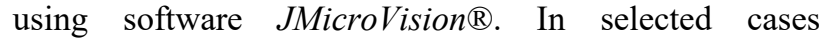
transcription of AtFLOT genes was analysed as well.

For high salinity, the seedlings were grown on $1 / 2 \mathrm{MS}$ solid medium containing $100 \mathrm{mM} \mathrm{NaCl}$. For gene transcription analysis 11-d-old seedlings were flooded with $150 \mathrm{mM} \mathrm{NaCl}$ dissolved in liquid $1 / 2 \mathrm{MS}$ medium for $3 \mathrm{~h}$. As a control seedlings were flooded with $1 / 2 \mathrm{MS}$ liquid medium.

For cold treatment, the seedlings were grown on $1 / 2 \mathrm{MS}$ solid medium at $14{ }^{\circ} \mathrm{C}$ and control seedlings at $22{ }^{\circ} \mathrm{C}$. For gene transcription analysis, 11-d-old seedlings were treated for $3 \mathrm{~h}$ by cultivation at $6{ }^{\circ} \mathrm{C}$ in darkness. Control seedlings were put into darkness for $3 \mathrm{~h}$ at $22{ }^{\circ} \mathrm{C}$.

For phosphate starvation experiment, the control seedlings were grown on modified half-strength Hoagland's medium (Hoagland et al. 1950) with $1 \%$ agar and the treated seedlings were grown on half-strength Hoagland's medium in which $\mathrm{NH}_{4} \mathrm{H}_{2} \mathrm{PO}_{4}$ was replaced with $\mathrm{NH}_{4} \mathrm{Cl}$. The medium was adjusted to $\mathrm{pH} 6.2$ with $\mathrm{NaOH}$.

For nitrogen starvation experiment, the control seedlings were grown on medium containing $1 \mathrm{mM}$ $\mathrm{KH}_{2} \mathrm{PO}_{4}, 25 \mu \mathrm{M} \mathrm{H} \mathrm{HO}_{3}, 2 \mu \mathrm{M} \mathrm{ZnSO}_{4}, 2 \mu \mathrm{M} \mathrm{MnSO}_{4}$, $0.5 \mathrm{mM} \mathrm{MgSO}_{4}, 20 \mu \mathrm{M}$ ferric citrate, $0.5 \mu \mathrm{M} \mathrm{CuSO}_{4}$, $0.5 \mu \mathrm{M} \mathrm{Na}_{2} \mathrm{MoO}_{4}, 1 \mathrm{mM} \mathrm{NH} \mathrm{NO}_{3}, 0.25 \mathrm{mM} \mathrm{CaSO} 4$, and $1 \%$ agar and the treated seedlings on the medium without $\mathrm{NH}_{4} \mathrm{NO}_{3}$.

Biotic stresses: The inoculation with Pseudomonas syringae pv. tomato DC3000 (Pst DC3000) was performed according to Katagiri et al. (2002) with slight modifications. In brief, bacteria were cultivated on the Luria-Bertani (LB) solid medium (with $1.2 \%, \mathrm{~m} / \mathrm{v}$, agar) containing rifampicin $\left(50 \mathrm{~g} \mathrm{dm}^{-3}\right)$ overnight. Bacteria were resuspended in $10 \mathrm{mM} \mathrm{MgCl}_{2}$ and a suspension was prepared to absorbance $\left(\mathrm{A}_{600}\right)=0.001$ for infiltration and $\mathrm{A}_{600}=0.2$ for dipping. For dipping inoculation suspension contained Silwet Star $(0.02 \%$, v/v, AgroBio, Opava, Czech Republic). Four-week-old plants were infiltrated with needleless syringe or dipped for $30 \mathrm{~s}$ in bacterial suspension. Nine discs ( $6 \mathrm{~mm}$ diameter) from three plants were collected as one sample of one genotype at 0 dpi and $3 \mathrm{dpi}$. The leaf discs were grounded in $10 \mathrm{mM} \mathrm{MgCl}_{2}$ and decimal dilution was performed. The colony forming units were counted. For gene transcription analysis, 4-week-old plants were infiltrated (using needleless syringe) with $P s t$ DC3000 for $24 \mathrm{~h}$, control plants were treated with $10 \mathrm{mM}$ $\mathrm{MgCl}_{2}$.

Four-week-old $A$. thaliana plants were treated with a 6- $\mathrm{mm}^{3}$ drops containing Botrytis cinerea BMM spores $\left(5 \times 10^{4}\right.$ spores $\left.\mathrm{cm}^{-3}\right)$ by applying one drop on one leaf, three leaves at similar developmental stage from one plant. The treated plants were transferred into the closed plastic box and were kept at low irradiance of $10-20 \mu \mathrm{mol} \mathrm{m}^{-2} \mathrm{~s}^{-1}$ ), a 16-h photoperiod and a temperature of $21{ }^{\circ} \mathrm{C}$ for $96 \mathrm{~h}$ post inoculation (hpi). For gene transcription analysis, 4-week-old plants were treated with Botrytis cinerea BMM spores $\left(5.10^{4}\right.$ spores $\left.\mathrm{cm}^{-3}\right)$ diluted in potato dextrose broth (PDB) liquid medium for $48 \mathrm{~h}$, control plants were treated with a drop of four times diluted PDB liquid medium.

Measurement of $\mathrm{H}_{2} \mathrm{O}_{2}$ production: $\mathrm{H}_{2} \mathrm{O}_{2}$ production was determined by the luminol-based assay as described in (Sasek et al. 2014). Discs, $3 \mathrm{~mm}$ in diameter, were cut from the fully developed leaves (two discs per leaf) of 4-weekold $A$. thaliana plants (three leaves per plant). Discs were incubated in white non-transparent 96-well plate (NUNC, Thermo Fisher Scientific, Waltham, MA, USA) in 0.15 $\mathrm{cm}^{3}$ of distilled water for $16 \mathrm{~h}$. Distilled water was replaced by $0.2 \mathrm{~cm}^{3}$ of reaction solution containing $17 \mu \mathrm{g} \mathrm{cm}^{-3}$ of luminol, $10 \mu \mathrm{g} \mathrm{cm}^{-3}$ of horseradish peroxidase (SigmaAldrich) and $100 \mathrm{nM}$ flg22 or $100 \mathrm{nM}$ elf18. The measurement was performed immediately after adding the flg22 with a luminometer (Tecan infinite F200, Männedorf, Switzerland) for a period of $45 \mathrm{~min}$.

Callose deposition in response to flg22: $A$. thaliana seedlings were treated with $1 \mu \mathrm{M}$ flg2 2 for $24 \mathrm{~h}$ at day 11 , the MS medium was replaced with fresh one with or without flg22. After $24 \mathrm{~h}$ the MS medium was replaced with ethanol:glacial acetic acid (3:1, v/v) until the seedlings were decolorized. The seedlings were then rehydrated in successive baths of $70 \%$ ethanol (at least $1 \mathrm{~h}), 50 \%$ ethanol (at least $1 \mathrm{~h}), 30 \%$ ethanol (at least $1 \mathrm{~h}$ ), and water (at least $2 \mathrm{~h}$ ). Leaves were then incubated in $0.01 \%(\mathrm{~m} / \mathrm{v})$ aniline blue in $150 \mathrm{mM} \mathrm{K}_{2} \mathrm{HPO}_{4}, \mathrm{pH} 9.5$, for 4 - $6 \mathrm{~h}$. Callose deposition was observed using fluorescence microscope AxioImager ApoTome2 (Carl Zeiss, Oberkochen, Germany) and the number of callose spots per $\mathrm{mm}^{2}$ were calculated using Fiji software (Schindelin et al. 2012). For gene transcription analysis, four-week-old A. thaliana plants were treated with $100 \mathrm{nM}$ flg 22 applied by needleless syringe infiltration for 1 and $4 \mathrm{~h}$. Infiltration with distilled water was used as a control.

Treatments with phytohormones: The seedlings were grown on $1 / 2 \mathrm{MS}$ medium containing $2 \mu \mathrm{M}$ ABA dissolved in $\mathrm{EtOH}(0.01 \%)$ and $1 \%$ agar, control seedlings grew on $1 / 2$ MS medium containing only EtOH. Medium was adjusted to $\mathrm{pH} 5.8$ with $\mathrm{KOH}$. Root length was analysed using software JMicroVision ${ }^{\circledR}$. For gene transcription 
analysis seedlings at day 11 were flooded with $100 \mu \mathrm{M}$ ABA in liquid $1 / 2 \mathrm{MS}$ medium for $3 \mathrm{~h}$ as a control seedlings were flooded with $0.1 \% \mathrm{EtOH}$ in liquid $1 / 2 \mathrm{MS}$ medium.

The 4-d-old seedlings grown on $1 / 2$ MS were transferred on $1 / 2$ MS medium containing $200 \mathrm{nM}$ NAA and length of the root was marked on the plate. The seedlings were scanned at day 4 after transfer and the length of primary

\section{Results}

Transcriptions of all three flotillin genes in 11-d-old A. thaliana seedlings exposed to $\mathrm{NaCl}$, cold, ABA, infection, and flg22 treatments were analysed. Flooding seedlings with $150 \mathrm{mM} \mathrm{NaCl}$ for $3 \mathrm{~h}$ significantly down regulated the transcription of AtFLOT1 and AtFLOT2 genes (Fig. 1A). Exposure of seedlings to $6{ }^{\circ} \mathrm{C}$ for $3 \mathrm{~h}$ increased the transcription of AtFLOT3 gene (Fig. 1B). After infiltration of 4-week-old $A$. thaliana with $100 \mathrm{nM}$ flg22, transcriptions of all AtFLOT genes increased 1 and $4 \mathrm{~h}$ after treatment (Fig. 1C) with strongly increased expressions of AtFLOT1 and AtFLOT3. Upregulation of AtFLOT3 was transient and after $4 \mathrm{~h}$ after returned to the basal level. The transcription of AtFLOT1 and AtFLOT2 genes further increased at $4 \mathrm{~h}$ after flg22 treatment (Fig. 1C). Interestingly, bacterial infection of plants with Pst DC3000 did not lead to the changes in transcription of AtFLOT genes (Fig. 1D), whereas infection with fungus $B$. cinerea induced transcription of AtFLOT1 and AtFLOT3 (Fig. 1E). Treatment with ABA altered the transription of all three AtFLOTs, however, the changes were not significant due to high variability of obtained data (Fig. $1 F$ ). Overall, transcription of at least one AtFLOT gene was significantly changed under 4 from 6 tested stress factors which strongly suggests involvement of AtFLOTs in response to stresses.

Following transcriptomic analysis, T-DNA knock-out single mutants available from public seeds depositories for each flotillin gene were used for phenotype analysis. These mutants do not transcribe particular AtFLOT genes (Fig. 1 Suppl.). We measured root growth of WT and mutant plants exposed to high salinity (100 mM NaCl; Fig. $2 A)$, cold $\left(14^{\circ} \mathrm{C}\right.$; Fig. $2 B$ ), and phosphate (Fig. $2 C$ ) and nitrogen starvation (Fig. 2D). Seedlings were exposed to these stresses for $7 \mathrm{~d}$. In contrast to transcriptomic analysis, comparison of flotillin mutant lines with their background genotype did not reveal any significant changes in root length and other noticeable morphological changes. These results indicate that any of single AtFLOT gene does not play particular role in acclimation to all tested abiotic stresses.

Furthermore, we focused on the role of the single flotillin gene in response to biotic stresses. Transcriptional analysis showed changes of AtFLOT gene expression in response to flg22 and $B$. cinerea (Fig. $1 C, E$ ). We measured $\mathrm{H}_{2} \mathrm{O}_{2}$ production upon microbe-associated molecular patterns (MAMP) treatment (Fig. $3 A$ ) since ROS burst is root was measured using software Fiji (Schindelin et al. 2012).

Statistical analysis: If not mentioned otherwise, twotailed Student's $t$-test was used for statistical evaluation and statistical significance was assigned to difference with $P$ values $<0.01$.

well described immediate and massive response to MAMPs (Smith et al. 2014). For Atflot1 and Atflot3 mutants we used flg22 as MAMP. Because Atflot2 mutant has a genetic background Ws-4 which lacks the flagellin-

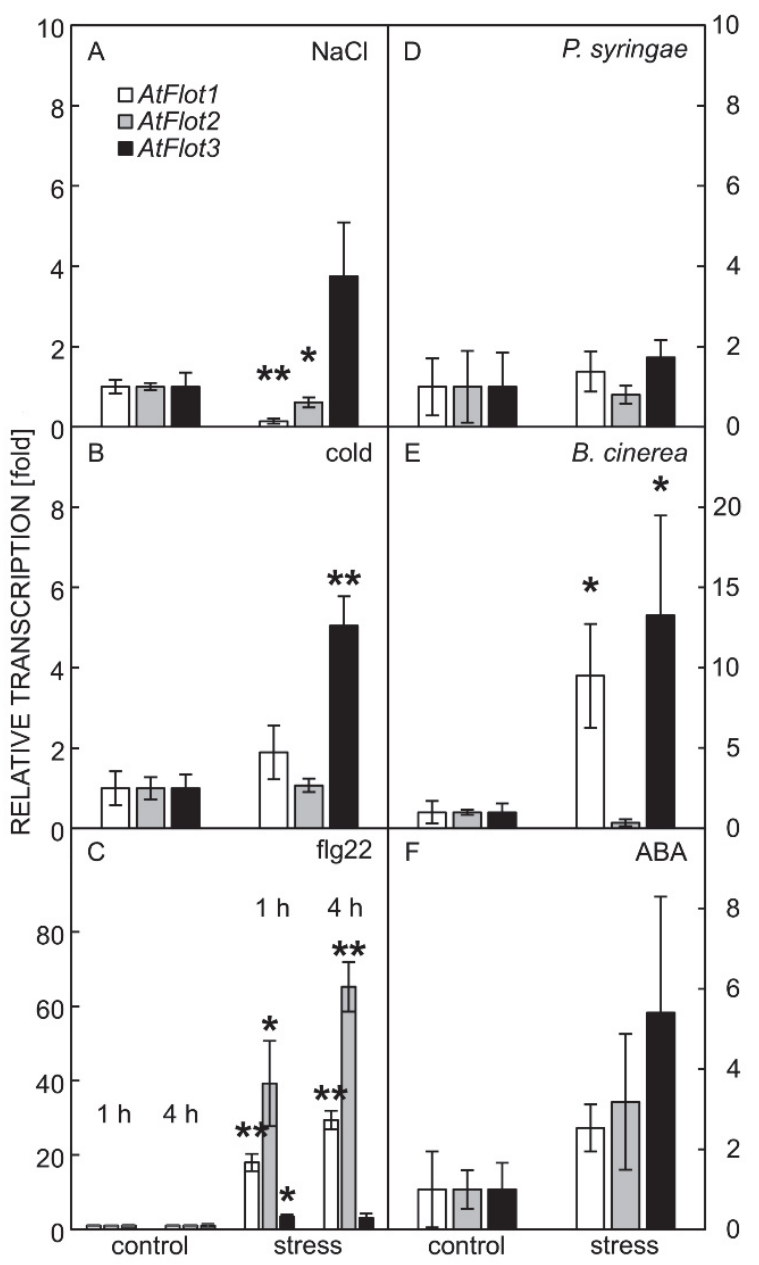

Fig. 1. Transcription analysis of AtFLOT genes in response to different stresses. Arabidopsis thaliana seedlings were treated at day 11 with $150 \mathrm{mM} \mathrm{NaCl}$ for $3 \mathrm{~h}(A), 6{ }^{\circ} \mathrm{C}$ for $3 \mathrm{~h}(B), 100 \mathrm{nM}$ flg22 for 1 and $4 \mathrm{~h}(C)$, infiltration with Pst DC3000 for $24 \mathrm{~h}(D)$, inoculation with Botrytis cinerea BMM spores for $48 \mathrm{~h}(E)$, and $100 \mu \mathrm{M}$ ABA for $3 \mathrm{~h}(F)$. Means $\pm \mathrm{SE}, n=3$ to 4 , asterisks indicate statistically significant differences compared to the corresponding control $(*-\mathrm{P}<0.05, * *-\mathrm{P}<0.01$, Student's $t$-test). Transcription was normalized to a reference gene TIP41-like. 
sensitive 2 (FLS2) receptor for flg22 (Zipfel et al. 2004), we used elf18 (Lu et al. 2009). However, none of the mutants had affected ROS production in response to MAMPs (Fig. 3A). Furthermore we tested the resistance of $A$. thaliana WT and mutant plants toward the infection with Pst DC3000, which represent model pathosystem in the studies of plant-bacteria interactions (Xin et al. 2018).

Here we used two different experimental approaches: infiltration with needleless syringe (Fig. $3 B$ ) and flooding of plant rosettes in bacterial suspension (Fig. 2 Suppl.). In both setups, no differences in the number of bacteria were found in the mutant line in comparison to the controls. Not surprisingly, the genotypes with Ws-4 background were more susceptible to Pst DC3000 compared to Col-0 background genotypes (Figs. 3 and 2 Suppl.). As the AtFLOT1 and AtFLOT3 transcription was induced in response to $B$. cinerea, we tested if these mutants would have altered resistance to this necrotrophic fungus. However, we did not see any significant difference between infected mutant and control lines (Fig. 3C). Moreover, Ws-4 genotypes were more resistant to the infection in comparison with Col-0 genotypes (Fig. 3C).

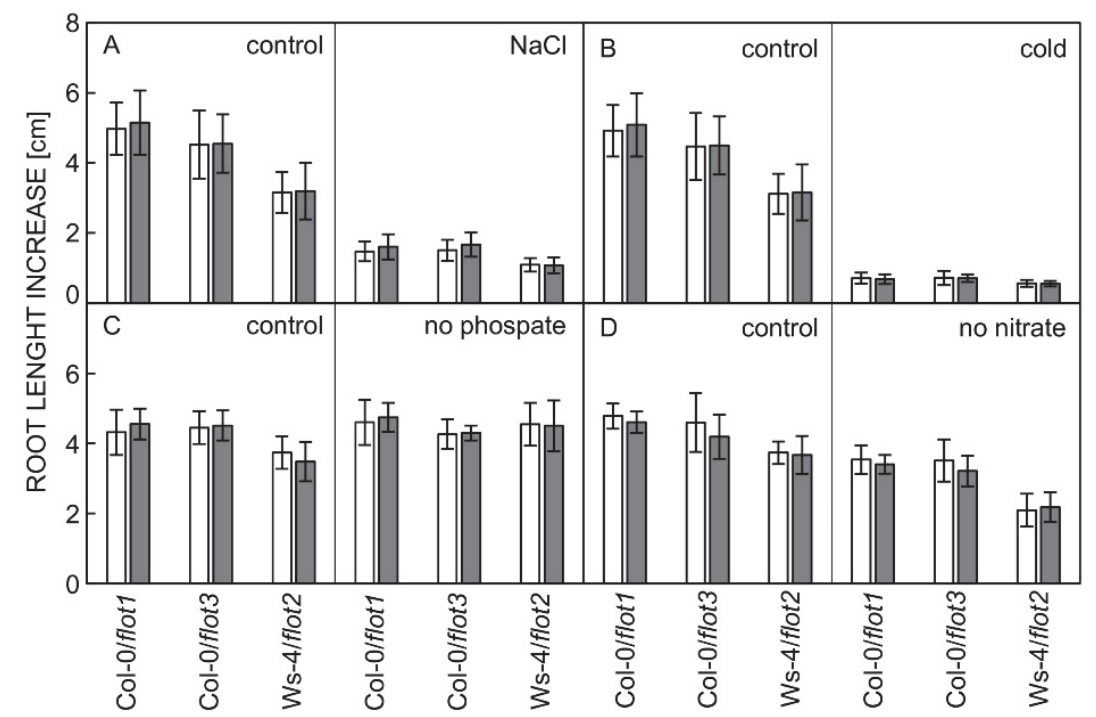

Fig. 2. Response of flotillin T-DNA A. thaliana mutants to abiotic stresses. Root growth of 12-d-old seedlings on the medium containing $100 \mathrm{mM} \mathrm{NaCl}$ for last $7 \mathrm{~d}(A)$. Root growth of 12-d-old seedlings $14{ }^{\circ} \mathrm{C}$ for last $7 \mathrm{~d}(B)$. Root growth of 12-d-old seedlings on the medium without phosphate for last $7 \mathrm{~d}(C)$. Root growth of 12-d-old seedlings medium without nitrogen for last $7 \mathrm{~d}(D)$. White bars represent WT, grey bars represent Atflot mutant. Means \pm SDs, $n=17$ to $20(P<0.01$, Student's $t$-test $)$

$\mathrm{Yu}$ et al. (2017) showed decreased callose accumulation in response to flg22 in seedlings of their Atflot1 knock-down mutant. Therefore, we measured callose accumulation in our Atflot 1 knock-out mutant, but we did not see the difference compared to the control line (Fig. $3 D$ ). Despite the transcriptional changes in response to biotic stress, we did not reveal the crucial role of particular AtFLOT gene under biotic stress conditions tested.

In sillico transcriptomic analysis showed trans- criptional changes of AtFLOT genes in response to phytohormones ABA and auxin (Daněk et al. 2016). Although our transcription analysis after treatment with ABA did not confirm microarray data, we performed the root growth assays with seedlings of all three knock-out mutants where we measured the root growth in presence of $100 \mu \mathrm{M}$ ABA or $200 \mathrm{nM}$ NAA. The root growth of Atflot mutants was similar to control lines (Fig. 4A,B).

\section{Discussion}

In our work we focused on the possible role of flotillins in response to different type of stresses in $A$. thaliana. The available transcriptomic microarray data indicated a possible involvement in response to abiotic and biotic stresses (Daněk et al. 2016). In terms of abiotic stress, here we show that transcription of AtFLOT genes is altered in early response (after $3 \mathrm{~h}$ ) to high salinity leading to the inhibition of AtFLOT1 and AtFLOT2 (Fig. 1A) and exposure to cold leading to the induction of AtFLOT3 transcription (Fig. 1B). In the case of biotic stress, the transcriptional changes were more robust compared to changes under abiotic stresses. In four-week-old A. thaliana the transcription of AtFLOT1 and AtFLOT2 was increased in response to flg22 (Fig. 1C) and transcription of AtFLOT1 and AtFLOT3 was increased in response to the infection by $B$. cinerea (Fig. $1 E$ ). The transcription profile upon $B$. cinerea treatment is interesting because under other tested conditions (high 
salinity, cold, flg22), mainly AtFLOT1 and AtFLOT2 share similar transcriptional pattern. This is not surprising with respect to the fact that they share $94 \%$ sequence similarity and therefore they may function similarly as well. However, in response to $B$. cinerea, the transcription of AtFLOT1 and AtFLOT3 was induced as opposed to the transcription of AtFLOT2 which remained stable (Fig. 1E). Hence, our results imply functional redundancy of $A t$ FLOT1 and $A t$ FLOT2 but only in some cases. Results from our transcriptomic analysis do not correspond in all cases with publicly available microarray data. Pst DC3000 infection did not affect transcription (Fig. 1D) and treatment with ABA did not show significant transcription changes due to very high variability of measurements (Fig. $1 F$ ). $\mathrm{NaCl}$ treatment had the opposite effect than it was revealed with microarray experiments, inhibition of transcription (Fig. 1A). However cold stress, flg22 treatment, and $B$. cinerea infection had similar effect on the AtFLOTs transcription as was found in database (Daněk et al. 2016). These discrepancies may be explained by slightly different conditions used in particular experiments. It must be noted as well that set up of microarray experiments does not allow discrimination between expressions of AtFLOT1 and AtFLOT2.
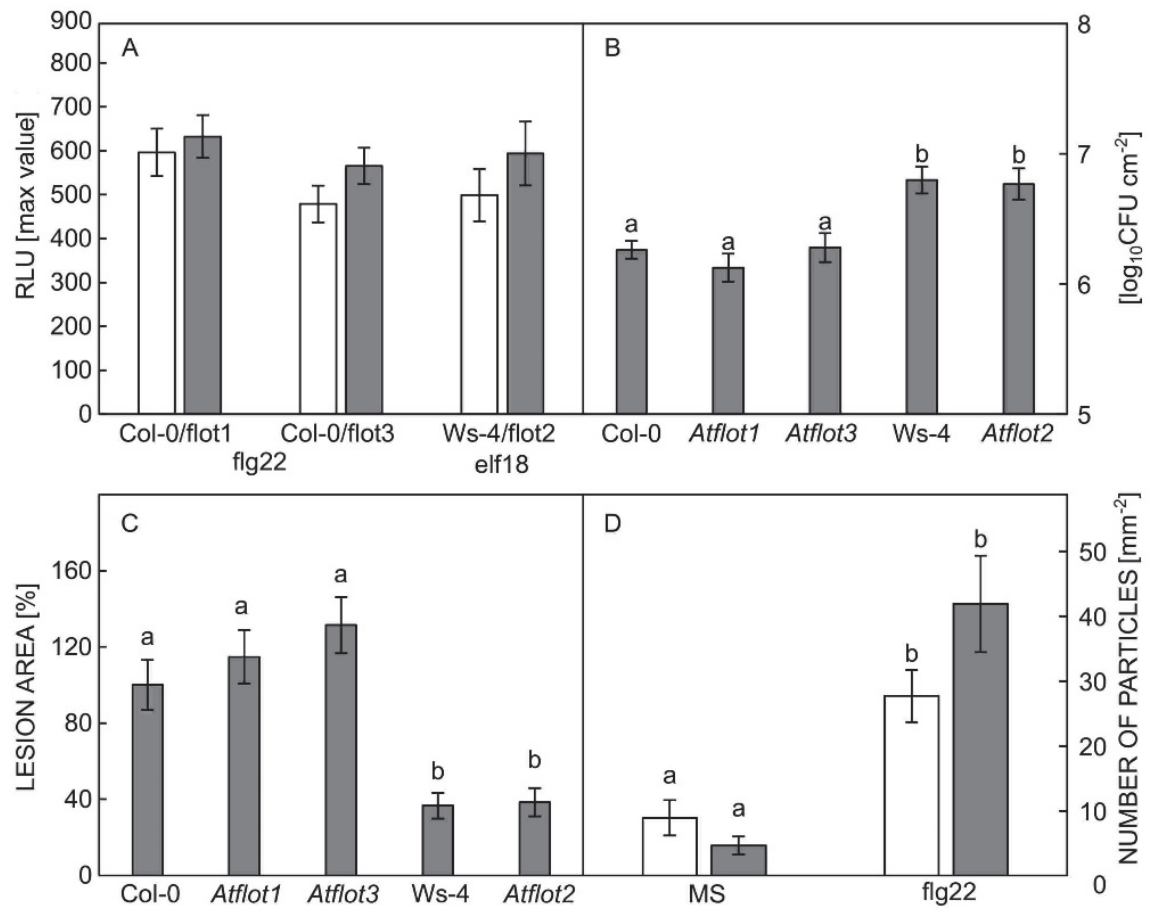

Fig. 3. Response of flotillin T-DNA $A$. thaliana mutants to biotic stresses. $A$ - Maximum luminescence induced by $100 \mathrm{nM}$ flg22 for Atflot 1 and Atflot 3 or $100 \mathrm{nM}$ elf1 8 for Atflot 2 in leaf discs from 4-week-old plants (RLU - relative luminiscence unit). B - Pst DC3000 was infiltrated into leaves of 4-week-old plants. Values are demonstrated in $\log _{10}$ scale of colony forming units (CFU). $C$ - Lesions on 4-week-old $A$. thaliana leaves infected with Botrytis cinerea BMM for 96 h. D - Callose deposition in 10-d-old seedlings of $A$. thaliana treated with $1 \mu \mathrm{M}$ flg22 for $24 \mathrm{~h}$ (MS - control). In $A$ and $D$, white bars represent WT, grey bars represent Atflot mutant. Means $\pm \mathrm{SEs}$, $n=12$ for $A, C$ and 6 for $B, D)$. Different letters indicate significant differences between the samples $(P<0.01$, Student's $t$-test $)$.

The second goal of the work was to investigate direct involvement of a particular AtFLOT gene in response to stresses. For that purpose, we used T-DNA knock-out mutants for every single AtFLOT gene. The proper characterisation of the mutants is the critical point. Li et al. (2012) showed that some of AtFLOT1 T-DNA insertion mutant lines had similar AtFLOT1 expression as WT or even over-expression. Here we used different T-DNA line of Atflot1 than Li et al. (2012) and also T-DNA mutants of Atflot2 and Atflot3 (Fig. 1 Suppl.). Obtained T-DNA lines did not show any transcription of particular AtFLOT genes (Fig. 1 Suppl.). We used above mentioned mutants for the phenotypic analysis in response to abiotic and biotic stress and for the treatment with phytohormones ABA and NAA.
According to best of our knowledge, no screening study of the involvement of flotillins in abiotic stresses exists until now. In silico AtFLOTs transcriptional data, as well as our results, indicated involvement of flotillins in response to abiotic stresses. Moreover, in yeasts and mammals flotillins play a role in endocytosis (Otto et al. 2011). Similar role was suggested for AtFLOT1. In specific conditions, it was shown that endocytosis of several plasma membrane (PM) proteins such as NADPH/respiratory burst oxidase protein D (RbohD), plasma membrane intrinsic protein 2 (PIP2;1), brassinosteroid insensitive 1 (BRI1) and ammonium transporter 1 (AMT1-3) is mainly dependent on clathrin mediated endocytosis but the role of microdomains and 
AtFLOT1 cannot be excluded (Hao et al. 2014, Li et al. 2012, Liu et al. 2009, Wang et al. 2013, Yu et al. 2017). The role of endocytosis in abiotic stresses was described as well. For example, salt stress increases PM endocytosis (Hamaji et al. 2009, Leshem et al. 2006) and cold stress inhibits intracellular trafficking (Shibasaki et al. 2009). We tested the root growth of mutant and WT plants under high salinity, cold, nitrogen starvation, and phosphate starvation. No differences between mutant and WT root growth under tested abiotic stresses were observed (Fig. 2). One of the possible explanations of this observation is gene redundancy of $A$. thaliana flotillins. This explanation is supported with our results and with results of Li et al. (2012) (for details see below) and it is reasonable especially in the case of AtFLOT1 and AtFLOT2. To reveal the role of redundant genes it is necessary to prepare the multiple Atflot knock-out mutant lines. CRISPR-Cas9 methodology would be a method of choice because AtFLOT1 and AtFLOT2 are in linkage and therefore it is not possible to obtain double mutant by crossing. Also, another experimental design should be considered.

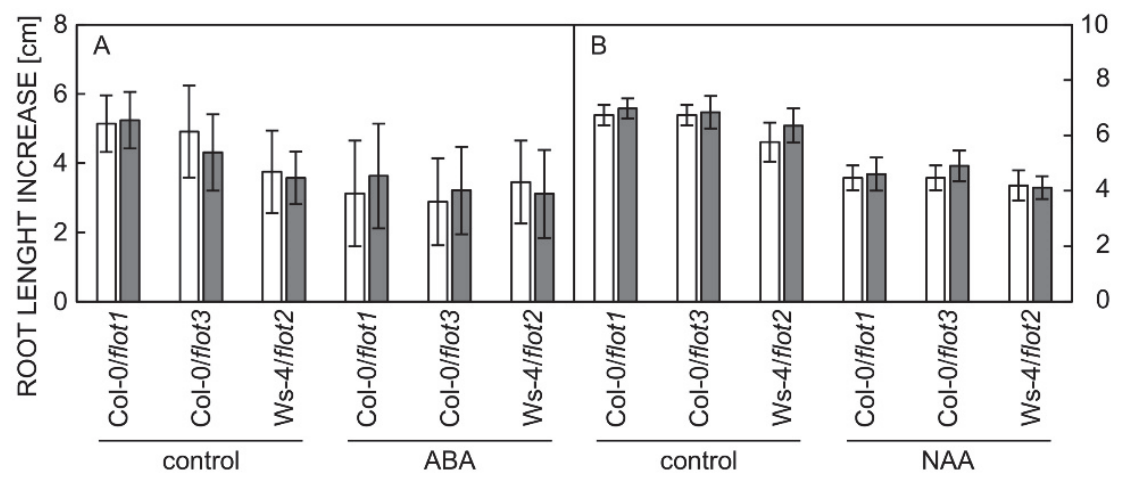

Fig. 4. Response of flotillin T-DNA $A$. thaliana mutants to phytohormones. $A$ - The root growth of 12-d-old seedlings on the medium containing $2 \mu \mathrm{M}$ ABA for last $7 \mathrm{~d}$. $B$ - The root growth of 8-d-old seedlings on the media containing $200 \mathrm{nM}$ NAA for last $4 \mathrm{~d}$. White bars represent WT, grey bars represent Atflot mutant. Means $\pm \mathrm{SD}, n=11$ to 30 .

In contrast to abiotic stresses, data showing the involvement of flotillins in biotic stresses already exist. The critical role of flotillins was shown in Medicago truncatula in response to symbiotic rhizobial infection. In $M$. truncatula seven flotillin-genes are recognised. Using silenced mutants in MtFLOT2 and MtFLOT4 it was approved that they are required for host derived infection threads and nodule formation (Haney et al. 2010). We tested T-DNA Atflot lines in response to Pst DC3000 and $B$. cinerea. However, we did not observe any difference between WT and T-DNA lines after Pst DC3000 and $B$. cinerea infection. The only differences we observed between the background genotypes Col-0 and Ws-4. Plants with Col-0 background were more resistant against Pst DC3000 and interestingly, more susceptible to $B$. cinerea in comparison to plants with $\mathrm{Ws}-4$ background (Fig. 3B,C). However, a molecular background of this phenomenon is not known.

Upregulation of AtFLOT gene transcription after flg22 treatment was shown (Daněk et al. 2016, Millet et al. 2010). In agreement, we observed increased transcription of AtFLOT genes after treatment with flg22. It is known that flg22 treatment results in higher ROS production and callose deposition (Denoux et al. 2008). We studied transient ROS production in response to treatment with flg22 in our T-DNA mutant lines and we did not observe any difference between flotillin knock-out mutant and WT plants (unpublished results). Also, we studied callose deposition in our T-DNA mutant lines and we did not observe any difference between flotillin knock-out mutant and WT plants. In contrary, Yu et al. (2017) showed that knock-down mutant of Atflot1 has decreased callose deposition in response to flg22. This contradiction may be explained by the downregulation of transcription of both AtFLOT1 and AtFLOT2. Yu et al. (2017) used in their study amiRNAi lines. The same lines were used by $\mathrm{Li}$ et al. (2012). Besides down-regulation of AtFLOT1, three from their four amiRNAi lines exhibited also downregulation of AtFLOT2 (Li et al. 2012). Li et al. (2012) also described growth inhibition of AmiRNAflotl line, however, we did not observe root growth retardation of our T-DNA AtFLOT1 line. These indicate that for observed decrease in callose deposition in response to flg22 and also the growth inhibition, both AtFLOT1 and AtFLOT2 are responsible.

Phytohormones play indispensable roles in plant growth and development and in response to both biotic and abiotic stresses (Santner et al. 2009, Denance et al. 2013, Janda and Ruelland 2015). Based on available transcriptomic data we were focused on the role of flotillins in response to ABA and NAA treatments. As for the biotic and abiotic stresses the role of endocytosis and microdomains in ABA and auxin mediated events are demonstrated. For example, auxin transporter PIN1 is present in microdomains (Titapiwatanakun et al. 2009) and polar distribution of auxin transporters is dependent on clathrin mediated endocytosis (Kitakura et al. 2011). ABA triggers the selective endocytosis of $A$. thaliana potassium 
channel KAT1 and its recycling to the PM in epidermal and guard cells (Sutter et al. 2007). As for the response to abiotic stress we analysed the effect of ABA and NAA to the root growth of Atflot T-DNA mutant lines. However, we did not observe any difference between T-DNA flotillin mutants and WT plants (Fig. 4).

We are aware that interpretation of the data obtained by the application of just one T-DNA mutated allele might be risky. Re-evaluation of function of abp 1-1 mutant could serve as an example of such situation (Dai et al. 2015, Enders et al. 2015, Michalko et al. 2015). Also the recent controversy dealing with commonly used Syngenta Arabidopsis insertion lines (SAIL) seeds stock with qrt1 background serves as highly important warning (Nikoronova et al. 2018). However, we believe that our results are not misinterpreted. In our study we did not use seeds from SAIL stock. Moreover, unlike in the above mentioned studies, we do not show an effect of T-DNA insertion into flotillin genes and therefore it is not necessary to consider additional T-DNA insertions.

In conclusion our transcriptomic analyses showed altered transcription of AtFLOT genes in response to both biotic and abiotic stresses. We obtained set of T-DNA single mutants which do not transcribe particular AtFLOT genes and we used them to screen involvement of single flotillin genes in response to broad spectrum of stresses. Our data showed that single flotillin genes are not the crucial components of $A$. thaliana response reactions to all stress conditions tested. The explanation could be the functional redundancy between AtFLOTs. Flotillins most probably act through the interaction with other proteins, thus their high sequence similarity may explain their redundancy. Creation of multiple knock-out lines will be necessary for further studies.

\section{References}

Bucherl, C. A., Jarsch, I. K., Schudoma, C., Segonzac, C., Mbengue, M., Robatzek, S., MacLean, D., Ott, T., Zipfel, C.: Plant immune and growth receptors share common signalling components but localise to distinct plasma membrane nanodomains. - eLife 6: e25114, 2017.

Daněk, M., Valentová, O., Martinec, J.: Flotillins, erlins, and hirs: from animal base camp to plant new horizons. - Crit. Rev. Plant Sci. 35: 191-214, 2016.

Denance, N., Sanchez-Vallet, A., Goffner, D., Molina, A.: Disease resistance or growth: the role of plant hormones in balancing immune responses and fitness costs. - Front. Plant Sci. 4: 155, 2013.

Denoux, C., Galletti, R., Mammarella, N., Gopalan, S., Werck, D., De Lorenzo, G., Ferrari, S., Ausubel, F. M., Dewdney, J.: Activation of defense response pathways by OGs and Flg22 elicitors in Arabidopsis seedlings. - Mol. Plants 1: 423-445, 2008.

Dai, X., Zhang, Y., Zhang, D., Chen, J., Gao, X., Estelle, M., Zhao, Y.: Embryonic lethality of Arabidopsis abp1-1 is caused by deletion of the adjacent $B S M$ gene. - Nat. Plants 1: pii: $15183,2015$.

Di, C., Xu, W., Su, Z., Yuan, J. S.: Comparative genome analysis of $P H B$ gene family reveals deep evolutionary origins and diverse gene function. - BMC Bioinformatics 11(Suppl 6): S22, 2010.

Enders, T. A., Oh, S., Yang, Z. B., Montgomery, B. L., Strader, L. C.: Genome sequencing of Arabidopsis abpl-5 reveals second-site mutations that may affect phenotypes. - Plant Cell 27: 1820-1826, 2015.

Glebov, O.O., Bright, N A., Nichols, B.J.: Flotillin-1 defines a clathrin-independent endocytic pathway in mammalian cells. - Nat. Cell Biol 8: 46-54, 2006.

Hamaji, K., Nagira, M., Yoshida, K., Ohnishi, M., Oda, Y., Uemura, T., Goh, T., Sato, M.H., Morita, M.T., Tasaka, M., Hasezaw, S., Nakano, A., Hara-Nishimura, I., Maeshima, M., Fukaki, H., Mimura, T.: Dynamic aspects of ion accumulation by vesicle traffic under salt stress in Arabidopsis. - Plant Cell Physiol. 50: 2023-2033, 2009.

Haney, C.H., Long, S.R.: Plant flotillins are required for infection by nitrogen-fixing bacteria. - Proc. nat. Acad. Sci. USA 107:
478-483, 2010.

Hao, H., Fan, L., Chen, T., Li, R., Li, X., He, Q., Botella, M. A., Lin, J.: Clathrin and membrane microdomains cooperatively regulate RbohD dynamics and activity in Arabidopsis. - Plant Cell 26: 1729-1745, 2014.

Hoagland, H., Arnon, D.I.: The water-culture method for growing plants without soil. - Calif. Agr. Exp. Sta 347: 1-32, 1950.

Janda, M., Ruelland, E.: Magical mystery tour: salicylic acid signalling. - Environ.eExp. Bot. 114: 117-128, 2015.

Jarsch, I.K., Konrad, S.S., Stratil, T.F., Urbanus, S.L., Szymanski, W., Braun, P., Braun, K.H., Ott, T.: Plasma membranes are subcompartmentalized into a plethora of coexisting and diverse microdomains in Arabidopsis and Nicotiana benthamiana. - Plant Cell 26: 1698-1711, 2014.

Katagiri, F., Thilmony, R., He, S.Y.: The Arabidopsis thaliana Pseudomonas syringae interaction. - Arabidopsis Book 1: e0039, 2002

Kitakura, S., Vanneste, S., Robert, S., Lofke, C., Teichmann, T., Tanaka, H., Friml, J.: Clathrin mediates endocytosis and polar distribution of PIN auxin transporters in Arabidopsis. Plant Cell 23: 1920-1931, 2011.

Lefebvre, B., Furt, F., Hartmann, M. A., Michaelson, L. V., Carde, J. P., Sargueil-Boiron, F., Rossignol, M., Napier, J. A., Cullimore, J., Bessoule, J. J., Mongrand, S.: Characterization of lipid rafts from Medicago truncatula root plasma membranes: a proteomic study reveals the presence of a raftassociated redox system. - Plant Physio.1 144: 402-418, 2007.

Leshem, Y., Melamed-Book, N., Cagnac, O., Ronen, G., Nishri, Y., Solomon, M., Cohen, G., Levine, A.: Suppression of Arabidopsis vesicle-SNARE expression inhibited fusion of $\mathrm{H}_{2} \mathrm{O}_{2}$-containing vesicles with tonoplast and increased salt tolerance. - Proc. nat. Acad. Sci. USA 103: 18008-18013, 2006.

Li, R., Liu, P., Wan, Y., Chen, T., Wang, Q., Mettbach, U., Baluska, F., Samaj, J., Fang, X., Lucas, W. J., Lin, J.: A membrane microdomain-associated protein, Arabidopsis Flot1, is involved in a clathrin-independent endocytic pathway and is required for seedling development. - Plant Cell 24: 2105-2122, 2012. 
Liu, P., Li, R. L., Zhang, L., Wang, Q.L., Niehaus, K., Baluska, F., Samaj, J., Lin, J.X.: Lipid microdomain polarization is required for NADPH oxidase-dependent ROS signaling in Picea meyeri pollen tube tip growth. - Plant J. 60: 303-313, 2009.

Lu, X., Tintor, N., Mentzel, T., Kombrink, E., Boller, T., Robatzek, S., Schulze-Lefert, P., Saijo, Y.: Uncoupling of sustained MAMP receptor signaling from early outputs in an Arabidopsis endoplasmic reticulum glucosidase II allele. Proc. natl.Acad. Sci. USA 106: 22522-22527, 2009.

Michalko, J., Dravecka, M., Bollenbach, T., Friml, J.: Embryolethal phenotypes in early abpl mutants are due to disruption of the neighboring BSM gene. - F1000Res 4: 1104, 2015.

Millet, Y.A., Danna, C.H., Clay, N.K., Songnuan, W., Simon, M.D., Werck-Reichhart, D., Ausubel, F M.: Innate immune responses activated in Arabidopsis roots by microbeassociated molecular patterns. - Plant Cell 22: 973-990, 2010.

Nikonorova, N., Yue, K., Beeckman, T., De Smet, I.: Arabidopsis research requires a critical re-evaluation of genetic tools. - J. exp. Bot. 15: 3541-3544, 2018.

Ott, T.: Membrane nanodomains and microdomains in plantmicrobe interactions. - Curr. Opin. Plant Biol. 40: 82-88, 2017.

Otto, G.P., Nichols, B.J.: The roles of flotillin microdomains endocytosis and beyond. - J. cell. Sci. 124: 3933-3940, 2011.

Santner, A., Estelle, M.: Recent advances and emerging trends in plant hormone signalling. - Nature 459: 1071-1078, 2009.

Sasek, V., Janda, M., Delage, E., Puyaubert, J., Guivarc'h, A., López Maseda, E., Dobrev, P.I., Caius, J., Bóka, K., Valentová, O., Burketová, L., Zachowski, A., Ruelland, E.: Constitutive salicylic acid accumulation in pi4kIIIbeta1beta2 Arabidopsis plants stunts rosette but not root growth. - New Phytol 203: 805-816, 2014.

Sekeres, J., Pleskot, R., Pejchar, P., Zarsky, V., Potocky, M.: The song of lipids and proteins: dynamic lipid-protein interfaces in the regulation of plant cell polarity at different scales. - J. exp. Bot. 66: 1587-1598, 2015.

Shibasaki, K., Uemura, M., Tsurumi, S., Rahman, A.: Auxin response in Arabidopsis under cold stress: underlying molecular mechanisms. - Plant Cell 21: 3823-3838, 2009.

Schindelin, J., Arganda-Carreras, I., Frise, E., Kaynig, V.,
Longair, M., Pietzsch, T., Preibisch, S., Rueden, C., Saalfeld, S., Schmid, B., Tinevez, J.Y., White, D.J., Hartenstein, V., Eliceiri, K., Tomancak, P., Cardona, A.: Fiji: an open-source platform for biological-image analysis. - Nat. Methods 9: 676-682, 2012.

Smith, J.M., Heese, A.: Rapid bioassay to measure early reactive oxygen species production in Arabidopsis leaf tissue in response to living Pseudomonas syringae. - Plant Methods 10: $6,2014$.

Sutter, J. U., Sieben, C., Hartel, A., Eisenach, C., Thiel, G., Blatt, M.R.: Abscisic acid triggers the endocytosis of the Arabidopsis KAT1 $\mathrm{K}+$ channel and its recycling to the plasma membrane. - Curr. Biol. 17: 1396-1402, 2007.

Titapiwatanakun, B., Blakeslee, J.J., Bandyopadhyay, A., Yang, H., Mravec, J., Sauer, M., Cheng, Y., Adamec, J., Nagashima, A., Geisler, M., Sakai, T., Friml, J., Peer ,W.A., Murphy, A.S.: ABCB19/PGP19 stabilises PIN1 in membrane microdomains in Arabidopsis. - Plant J. 57: 27 44, 2009.

Wang, L., Li, H., Lv, X.Q., Chen, T., Li, R.L., Xue, Y.Q., Jiang, J.J., Jin, B., Baluska, F., Samaj, J., Wang, X.L., Lin, J.X.: Spatio-temporal dynamics of the BRI1 receptor and its regulation by membrane microdomains in living Arabidopsis cells. - Mol. Plants 8: 1334-1349, 2015.

Wang, Q., Zhao, Y., Luo, W., Li, R., He, Q., Fang, X., Michele, R. D., Ast, C., Von Wiren, N., Lin, J.: Single-particle analysis reveals shutoff control of the Arabidopsis ammonium transporter AMT1;3 by clustering and internalization. - Proc. nat. Acad. Sci. USA 110: 13204-13209, 2013.

Xin, X.F., Kvitko, B., He, S.Y.: Pseudomonas syringae: what it takes to be a pathogen. - Nat. Rev. Microbiol. 16: 316-328, 2018.

Yu, M., Liu, H., Dong, Z., Xiao, J., Su, B., Fan, L., Komis, G., Samaj, J., Lin, J., Li, R.: The dynamics and endocytosis of Flot1 protein in response to flg22 in Arabidopsis. - J. Plant Physio.1 215: 73-84, 2017.

Zipfel, C., Robatzek, S., Navarro, L., Oakeley, E. J., Jones, J. D., Felix, G., Boller, T.: Bacterial disease resistance in Arabidopsis through flagellin perception. - Nature 428: 764 767, 2004. 